\title{
Influência dos factores financeiros no cumprimento da medicação
}

Ângela Chin,* Mariana Alves,* Nuno Martins,* Cátia Pedro,* Ana Ferreira,* Carolina Barbeiro,* Mafalda Mota,* Armando Brito de Sá**

\section{RESUMO}

Introdução: A situação financeira da população é um dos elementos que afecta o cumprimento da terapêutica médica.

Objectivos: Avaliar o cumprimento da medicação regular, as diferentes formas de não cumprimento por motivos financeiros e a transmissão dessa informação ao médico, assim como avaliar estratégias facilitadoras do cumprimento da medicação.

Tipo de Estudo: Observacional, descritivo e transversal.

Local: Unidade de Saúde Familiar de Loures.

População: Utentes inscritos na Unidade de Saúde Familiar, com médico de família e idade acima dos 17 anos, em Abril de 2011.

Métodos: Amostra de conveniência de 227 indivíduos. Colheita de informação por questionário, onde se estudou o sexo; idade; e, relativamente aos últimos quatro meses, por motivos financeiros: espaçamento da toma; redução da dose; adiamento da compra; ausência da compra; informação ao médico do não cumprimento da medicação; não cumprimento da medicação por motivos não financeiros; pedido de prescrição de medicamentos mais baratos; pedido de apoio financeiro para a compra da medicação; abdicação da compra de outros bens para comprar medicação.

Resultados: Dos 227 inquiridos, $79 \%$ referiu não cumprir a medicação, dos quais $51 \%$ o fez por razões financeiras (31\% exclusivamente). O não cumprimento da medicação por motivos financeiros deveu-se, em $84 \%$ dos indivíduos, ao adiamento da compra da medicação, $46 \%$ à ausência da compra da mesma, $44 \%$ ao aumento do espaçamento entre tomas e $38 \%$ à diminuição da dose. Dos indivíduos que não cumpriram por motivos financeiros, apenas $30 \%$ informou o seu médico. Quanto a estratégias facilitadoras do cumprimento da medicação, $52 \%$ referiu ter pedido a prescrição de medicamentos mais baratos, $41 \%$ abdicou da compra de outros bens e $12 \%$ pediu apoio financeiro.

Conclusões: O não cumprimento da medicação por motivos financeiros foi relatada numa elevada percentagem de indivíduos, a maioria dos quais não revelou essa informação ao seu médico.

Palavras-chave: Medicação Crónica; Adesão; Factores Financeiros.

\section{INTRODUÇÃO}

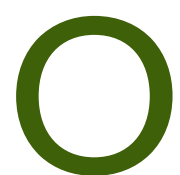

O cumprimento da terapêutica é uma temática complexa e multifactorial. ${ }^{1}$ É influenciada por factores directamente relacionados com o doente, pela respectiva medicação e também pela situação económica do país e das pessoas.

A identificação dos doentes que não aderem à terapêutica é difícil, mas essencial, para que se possa identificar e corrigir os seus motivos da não adesão, ${ }^{2,3}$ uma

*Alunos $6 .^{\circ}$ ano da Faculdade Medicina da Universidade de Lisboa

**Instituto de Medicina Preventiva, Faculdade de Medicina da Universidade de Lisboa vez que o não cumprimento da terapêutica é responsável pelo seu insucesso, pelo aumento dos custos com a saúde e pelo aumento da morbilidade e mortalidade. ${ }^{4-7}$

Alguns estudos referem a ausência de evidência entre os factores socio-económicos e o grau de adesão à terapêutica. ${ }^{1}$ No entanto, outros estudos internacionais referem que a subutilização medicamentosa por motivos financeiros varia entre $2 \%$ e $32 \% .^{5-11}$ Além disso, alguns autores referem estar a verificar-se um agravamento do problema pois, num estudo realizado com uma população diabética americana, observou-se uma redução da adesão à terapêutica por factores financei- 
ros, atingindo 8,7\% em 2003 e 10,7\% em $2007 .{ }^{12}$

Em Portugal, no contexto da situação económica dos últimos anos, com redução dos rendimentos, alteração do regime de comparticipação dos medicamentos e aumento da carga fiscal, os factores financeiros podem ter uma influência cada vez maior no cumprimento da terapêutica.

Contudo, a informação relativa à adesão à terapêutica no nosso país é escassa, sobretudo no tocante aos motivos financeiros. O primeiro estudo foi realizado por Cabral e Silva, tendo sido concluído que cerca de um terço dos doentes crónicos não comprou medicamentos por razões económicas. ${ }^{13}$

Entre os diversos métodos existentes para avaliar a adesão à terapêutica, os factores financeiros raramente são contemplados. No entanto, é possível encontrar na bibliografia algumas referências. No estudo de Delgado $\mathrm{AB}$ e Lima ML, um dos itens do questionário sobre adesão à terapêutica está associado às dificuldades financeiras: «Alguma vez interrompeu a terapêutica para a sua doença por ter deixado acabar os medicamentos?»14 Outros estudos utilizam questões como: «Você alguma vez saltou doses de medicamentos para que eles durem mais?»;"11 "Alguma vez, nos últimos dois anos, diminuiu a toma da medicação prescrita devido ao seu custo"; ${ }^{5}$ "Pensando especificamente na sua doença, quantas vezes, nos últimos 12 meses, efectuou o seguinte por motivos financeiros: tomou menos comprimidos ou uma dose menor, não comprou a medicação prescrita, adiou a compra da medicação, utilizou ervanárias ou vitaminas quando se sentiu doente em vez de tomar a medicação prescrita, tomou a medicação menos frequentemente do que o recomendado para adiar a renovação da medicação». ${ }^{2}$

No entanto, na nossa pesquisa bibliográfica, não encontrámos nenhum questionário português validado que tivesse como objectivo esta temática.

Este trabalho teve como objectivos estudar o cumprimento da medicação regular, as diferentes formas de não cumprimento por motivos financeiros e a transmissão dessa informação ao médico, e a identificação de estratégias facilitadoras do cumprimento da medicação.

\section{MÉTODOS}

Realizou-se um estudo observacional, descritivo e transversal, de uma amostra de conveniência da po- pulação que frequentou a Unidade de Saúde Familiar de Loures, em quatro dias consecutivos de Abril de 2011.

Como critérios de inclusão foram considerados: utentes de ambos os sexos; idade superior a 17 anos; existência de consentimento informado para entrar no estudo; e realização de medicação regular há pelo menos quatro meses.

Foram estudadas as seguintes variáveis: sexo; idade; espaçamento da toma por motivos financeiros; redução da dose por motivos financeiros; adiamento da compra por motivos financeiros; ausência da compra por motivos financeiros; informação ao médico do não cumprimento da medicação por motivos financeiros; não cumprimento da medicação por factores não financeiros; pedido de prescrição de medicamentos mais baratos; pedido de apoio financeiro para a compra da medicação; e abdicação de compra de outros bens para comprar medicação. Todas as variáveis foram operacionalizadas por resposta dicotómica simples (sim/não), com excepção do sexo e idade (inferior a 40 anos, entre 40 e 64 anos, igual ou superior a 65 anos).

Foi construído um questionário, o qual foi totalmente preenchido na sala de espera, antes da consulta médica. A maioria dos questionários foi preenchida pelos inquiridores, estudantes de medicina devidamente identificados, todos membros da equipa de investigação e conhecedores quer do projecto, quer do instrumento. Verificou-se o auto-preenchimento num reduzido número de situações, a pedido do próprio participante.

Foi considerado "Cumprimento da medicação», o facto de responder negativamente a todos os tópicos relacionados com a toma adequada da prescrição: espaçar a toma, reduzir a dose, adiar a compra e abdicar da compra da medicação. Foi considerado «Não cumprimento», a existência de resposta positiva a, pelo menos, um dos tópicos referidos.

Os dados obtidos foram analisados por estatística descritiva simples, utilizando Microsoft Office Excel 2007.

Este estudo respeitou os princípios da Declaração de Helsínquia modificada em Edimburgo (Outubro 2000), garantindo a confidencialidade e o anonimato acerca da identidade dos participantes, bem como a garantia da utilização dos seus dados apenas para fins estatísticos. 


\section{RESULTADOS}

Foram convidadas a participar no estudo 247 pessoas, sendo que 232 aceitaram responder ao questionário (taxa de respostas de 94\%). Destas, foram excluídas cinco por não terem realizado terapêutica regular desde o início de 2011. Foram assim incluídos 227 pacientes no estudo, dos quais quinze $(6,6 \%)$ fizeram o auto-preenchimento do questionário.

Nesta amostra, $152(67 \%)$ dos inquiridos eram do sexo feminino e 75 (33\%) do sexo masculino. Cinquenta participantes (22\%) tinham menos de 40 anos, 93 (41\%) entre 40 e 64 anos e 82 (36\%) mais de 64 anos.

Do questionário efectuado, foram obtidos os resultados apresentados no Quadro I.

Entre os inquiridos, $79 \%$ referiu não cumprir a medicação, dos quais $69 \%$ eram do sexo feminino. Tendo em conta a faixa etária, $23 \%$ tinham idade inferior a 40 anos, $39 \%$ entre 40 e 64 anos e $38 \%$ idade igual ou superior a 65 anos.

A partir da análise da Fig. 1, é possível constatar que $31 \%$ dos indivíduos que não cumpriram a medicação fizeram-no exclusivamente por factores financeiros, $36 \%$ exclusivamente por factores não financeiros, e $33 \%$ por ambos. Desta forma, no total, a motivação financeira foi referida por $51 \%$ dos inquiridos, dos quais a maioria era do sexo feminino e pertencia às faixas etárias acima dos 39 anos.

Em relação às formas de não cumprimento da medicação, estas consistiram em adiamento da compra (84\%), não aquisição da medicação (46\%), aumento do espaçamento entre tomas (44\%) e diminuição da dose (38\%) (Fig. 2).

Dos indivíduos que não cumpriram a medicação por motivos financeiros, $30 \%$ informou o seu médico, enquanto $70 \%$ não o fez.

Quanto a estratégias facilitadoras do cumprimento da medicação, $52 \%$ afirmaram ter pedido medicamentos mais baratos ao seu médico, $41 \%$ abdicaram da compra de outros bens e $12 \%$ pediram apoio financeiro para a compra de medicamentos. Dentro de cada um destes três grupos, a maioria dos inquiridos não cumpriu a medicação devido a razões financeiras (Fig. 3).

\begin{tabular}{|c|c|c|}
\hline \multirow[t]{3}{*}{ Pergunta } & \multicolumn{2}{|c|}{ Resposta* } \\
\hline & $\operatorname{sim}$ & não \\
\hline & $\mathrm{n}(\%)$ & $\mathrm{n}(\%)$ \\
\hline \multicolumn{3}{|l|}{ Alguma vez, por motivos financeiros: } \\
\hline $\begin{array}{l}\text { - aumentou o espaçamento entre as tomas de um medicamento para } \\
\text { diminuir a quantidade de vezes que o compra }\end{array}$ & $51(22,5)$ & $176(77,5)$ \\
\hline $\begin{array}{l}\text { - reduziu a dose de um medicamento para diminuir a quantidade de vezes } \\
\text { que o compra }\end{array}$ & $44(19,4)$ & $183(80,6)$ \\
\hline - adiou a compra de um medicamento & $97(42,7)$ & $130(57,3)$ \\
\hline - deixou de comprar um medicamento na farmácia & $53(23,3)$ & $174(76,7)$ \\
\hline $\begin{array}{l}\text { Alguma vez informou o seu médico que não cumpriu a medicação por } \\
\text { motivos financeiros }\end{array}$ & $36(15,9)$ & $191(84,1)$ \\
\hline Alguma vez não cumpriu a medicação por outras razões que não financeiras & $123(54,2)$ & $104(45,8)$ \\
\hline Alguma vez pediu ao médico para prescrever medicamentos mais baratos & $118(52,0)$ & $109(48,0)$ \\
\hline Alguma vez pediu apoio económico para comprar medicamentos** & $28(12,3)$ & $198(87,2)$ \\
\hline $\begin{array}{l}\text { Alguma vez deixou de comprar outros bens materiais para comprar } \\
\text { medicamentos por motivos financeiros }\end{array}$ & $94(41,4)$ & $133(58,6)$ \\
\hline
\end{tabular}

${ }^{*} \mathrm{n}=227{ }^{* *} \mathrm{n}=226$. 


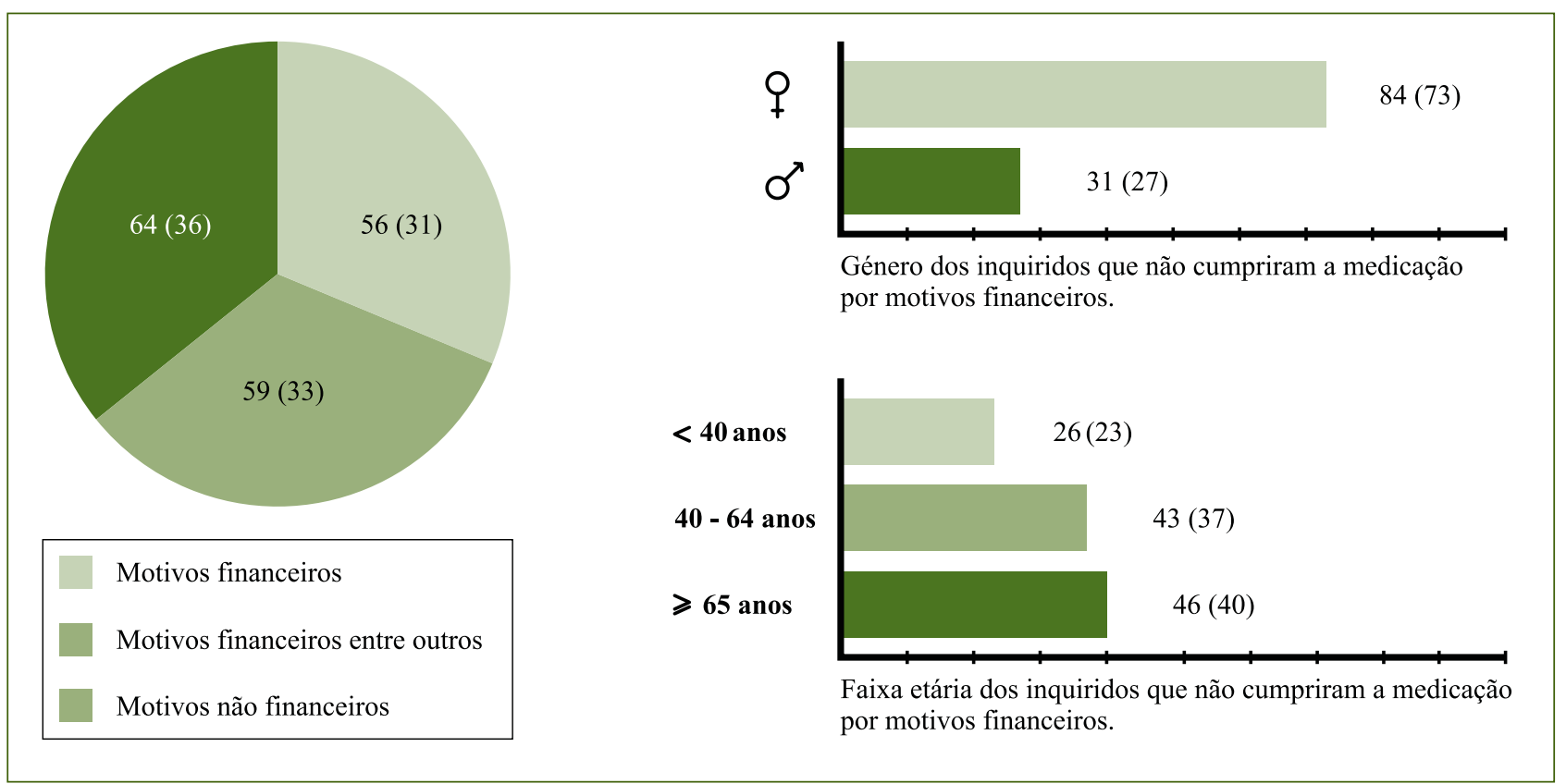

Figura 1. Motivos do não-cumprimento da medicação e a sua distribuição demográfica.

\section{DISCUSSÃO}

A utilização de um questionário no estudo da adesão à terapêutica é uma metodologia simples, eficaz e de baixo custo. ${ }^{1}$ Apesar de não ser validado, este reúne questões semelhantes a outras previamente utilizadas noutros estudos. ${ }^{1,2,5,9,11,14}$

A elevada taxa de resposta a este questionário apoia a fiabilidade dos resultados obtidos e demonstra a sua aplicabilidade. Esta é ainda facilitada pelo facto de incidir sobre uma temática actual e de interesse comum e pelo tipo de resposta dicotómica simples utilizada, embora possa estar associada a uma menor consistência interna.

Sendo esta amostra de conveniência, não pode ser extrapolada para a generalidade da população. Contudo, foi constituída predominantemente por indivíduos do sexo feminino e de grupos etários mais avançados, o que se aproxima da população habitual que recorre aos Centros de Saúde. ${ }^{15}$

De assinalar que a grande maioria dos questionários foi preenchida pelos inquiridores (apenas $6,6 \%$ foram auto-preenchidos), o que, tendo em conta o cariz particular e pessoal do tema abordado, poderá ter influenciado a resposta a algumas questões e assim, constitui uma limitação a este estudo. No entanto, ressalva-

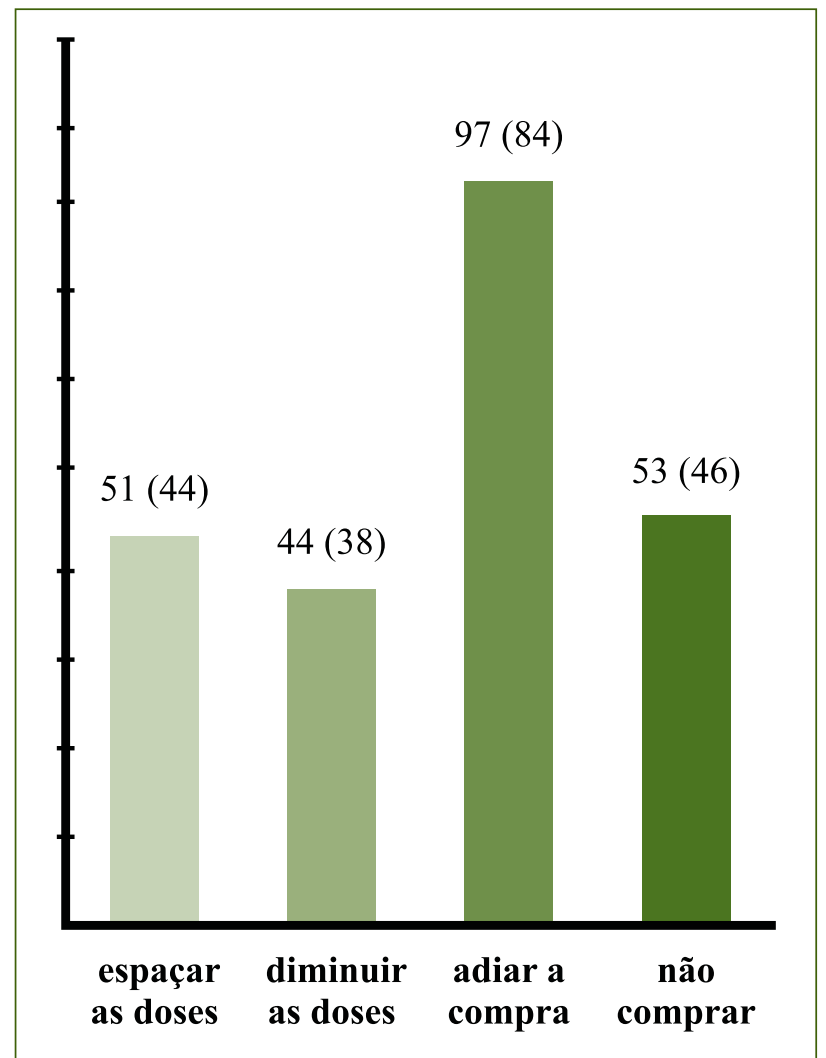

Figura 2. Diferentes formas de não-cumprimento da medicação por motivos financeiros. 


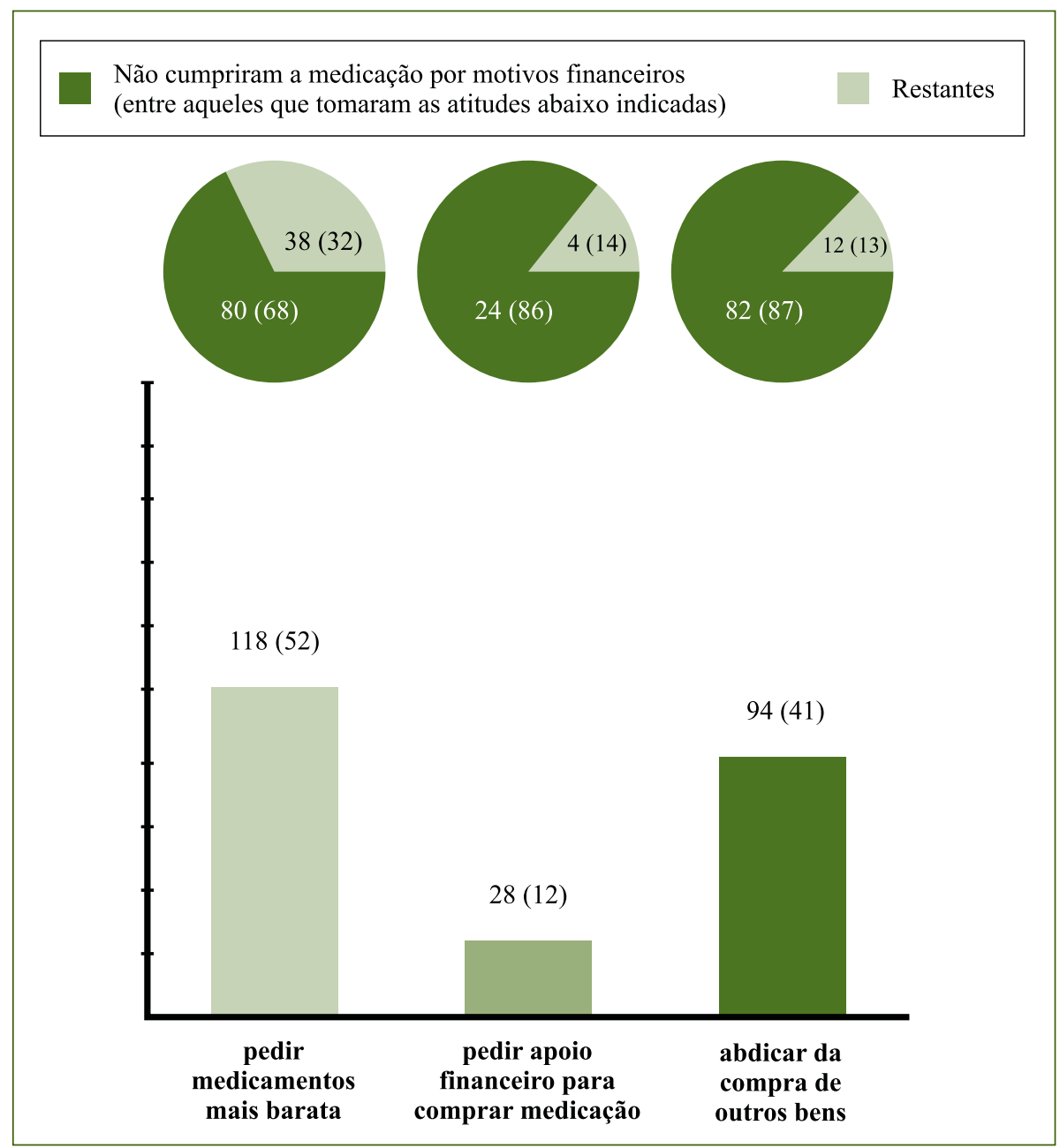

Figura 3. Estratégias facilitadoras do cumprimento da medicação e a sua relação com o não-cumprimento da mesma por motivos financeiros.

se que os inquiridores garantiram sempre o anonimato e a confidencialidade dos participantes ao longo de todo o processo.

Segundo os resultados obtidos, apenas $21 \%$ dos utentes cumpriu a medicação nos últimos quatro meses, o que é um valor inferior ao referido pela Organização Mundial da Saúde relativamente aos países desenvolvidos (50\%). ${ }^{4}$ Em 51\% dos casos, o não cumprimento deveu-se a motivos financeiros, um valor claramente superior ao previsto na literatura internacional, que varia entre $2 \%$ e $32 \% .^{5-11}$

Diferentes estratégias foram adoptadas para o não cumprimento da medicação devido a factores financeiros. A mais utilizada foi o adiamento da compra, re- ferida em $84 \%$ dos casos, ainda que estes dados devam ser analisados com cautela, pois o facto de adiar a compra não nos garante que o doente não tivesse ainda terapêutica em casa. No entanto, e perante a forma objectiva como a pergunta foi formulada, é de admitir que esta limitação não tenha um impacto relevante nos resultados obtidos.

Em segundo lugar, $46 \%$ dos indivíduos que não cumpriram a medicação por razões financeiras referiram já ter abdicado da compra da mesma. Este valor corresponde a $23 \%$ da população total estudada, o que está de acordo com a literatura: no estudo nacional de Cabral e Silva, $25,2 \%$ dos doentes crónicos abdicou da compra de medicamentos por não poder comportar os custos dos mesmos. ${ }^{13}$ No inquérito nacional americano realizado por Safran et al. aos beneficiários da Medicare com idade superior 65 anos, 23,3\% também não comprou a medicação pelos mesmos motivos. ${ }^{9}$

O espaçamento entre as tomas foi mencionado por $44 \%$ dos indivíduos que não cumpriram a medicação por motivos financeiros, o que corresponde a $22 \%$ da amostra inquirida, um valor superior aos $15,8 \%$ encontrados no estudo de Safran et al. previamente referido. ${ }^{9}$

Finalmente, $38 \%$ dos indivíduos que não cumpriram a medicação por motivos financeiros diminuíram a dose. Se for tida em conta toda a amostra inquirida, esta redução foi referida por $19 \%$ dos inquiridos, um valor próximo ao encontrado em vários estudos: no estudo de Briesacher et al. sobre a população diabética acima dos 65 anos, 19\% diminuiu a dose da medicação devido a factores financeiros ${ }^{16}$; no estudo de Piette em 
doentes crónicos adultos, $18 \%$ fez o mesmo 2 ; e no estudo de Safran et al. esta redução aconteceu em 12,4\%. ${ }^{9}$

No estudo de Briesacher et al. apenas 33\% dos doentes comunicou as alterações da medicação ao seu médico. ${ }^{16} \mathrm{Um}$ valor semelhante foi encontrado neste estudo, onde $30 \%$ dos que não cumpriram a terapêutica por motivos financeiros informaram o médico. Isto indica que, em $70 \%$ destas situações, os médicos podem não relacionar um possível insucesso da terapêutica com o não cumprimento da mesma, nem adaptar a terapêutica à situação financeira do doente.

Cinquenta e dois por cento dos inquiridos afirmaram ter pedido ao médico para lhes prescrever medicamentos mais baratos. Apesar de este pedido poder ter sido devido a uma preferência por medicamentos mais baratos e não indicar, necessariamente, uma dificuldade financeira no cumprimento da medicação, verificou-se que $68 \%$ desses doentes não cumpriu a medicação por razões financeiras.

Outro aspecto relevante é o facto de um maior número de pessoas $(41 \%)$ terem optado por deixar de comprar outros bens, como alimentos ou vestuário, do que terem pedido apoio financeiro $(12 \%)$ para comprar medicamentos. No estudo de Briesacher et al. menos inquiridos (20\%) abdicaram da compra de bens essenciais e $10 \%$ recorreram à ajuda de familiares. ${ }^{16}$ No estudo de Safran et al. foi encontrada uma percentagem ainda mais baixa de indivíduos que poupou em bens essenciais para comprar a medicação $(12,2 \%) .{ }^{9}$

Neste questionário, não foi analisada a relação da adesão à terapêutica com capacidade financeira dos inquiridos. Contudo, trata-se de um factor reconhecidamente relevante, ${ }^{17,18}$ pelo que ele deverá ser tido em conta em estudos futuros. Outros aspectos merecem atenção ulterior, nomeadamente quais os fármacos mais afectados pelas alterações efectuadas pelos doentes à medicação prescrita.

Concluindo, os resultados deste estudo apontam para uma importância relevante dos factores financeiros como causa do não cumprimento da medicação, o que era esperado. Porém, é de salientar a elevada percentagem de indivíduos que se incluiu neste grupo (51\%) e que não informou o seu médico (70\%). Uma vez que a má adesão à terapêutica está associada ao aumento dos custos directos para o controlo da doença $\mathrm{e}$ indirectos na saúde,$^{19} \mathrm{o}$ cumprimento da mesma deve ser incentivado, pois é tanto benéfico para o doente, como para o Sistema Nacional de Saúde.

\section{REFERÊNCIAS BIBLIOGRÁFICAS}

1. Osterberg L, Blaschke T. Adherence to medication. N Engl J Med 2005 Aug 4; 353 (5): 487-97.

2. Piette JD, Heisler $M$, Wagner TH. Cost-related medication underuse among chronically ill adults: the treatments people forgo, how often, and who is at risk. Am J Public Health 2004 Oct; 94 (10): 1782-7.

3. Sousa S, Pires A, Conceição C, Nascimento T, Grenha A, Braz L. Polimedicação em doentes idosos: adesão à terapêutica. Rev Port Clin Geral 2011 Mar-Abr; 27 (2): 176-82.

4. Sabaté E. Adherence to long-term therapies: evidence for action. Geneva: World Health Organization; 2003.

5. Steinman MA, Sands LP, Covinsky KE. Self-restriction of medication due to cost in seniors without prescription coverage: a national survey. J Gen Intern Med 2001 Dec; 16 (12): 793-9.

6. Kurlander JE, Kerr EA, Krein S, Heisler M, Piette JD. Cost-related nonadherence to medication among patients with diabetes and chronic pain: factors beyond finances. Diabetes Care 2009 Dec; 32 (12): 21438.

7. Rector TS, Venus PJ. Do drug benefits help Medicare beneficiaries afford prescribed drugs? Health Aff (Millwood) 2004 Jul-Aug; 23 (4): 213-22.

8. Piette JD, Wagner TH, Potter MB, Schillinger D. Health insurance status, cost-related medication underuse, and outcomes among diabetes patients in three systems of care. Med Care 2004 Feb; 42 (2): 102-9.

9. Safran DG, Neuman P, Schoen C, Kitchman MS, Wilson IB, Cooper B, et al. Prescription drug coverage and seniors: findings from a 2003 national survey. Health Aff (Millwood) 2005 Jan-Jun; Suppl Web Exclusives:W5-152-W5-166.

10. Mojtabai R, Olfson M. Medication costs, adherence, and health outcomes among Medicare beneficiaries. Health Aff (Millwood) 2003 Jul-Aug; 22 (4): 220-9.

11. Luz TC, de Loyola Filho Al, Lima-Costa MF. Estudo de base populacional da subutilização de medicamentos por motivos financeiros entre idosos na Região Metropolitana de Belo Horizonte, Minas Gerais, Brasil. Cad Saúde Pública 2009 Jul; 25 (7): 1578-86.

12. Chan M. Reducing cost-related medication nonadherence in patients with Diabetes. Drug Benefit Trends 2010; 22 (3): 67-71.

13. Cabral MV, Silva PA. Adesão à terapêutica em Portugal: atitudes e comportamentos da população portuguesa perante as prescrições médicas. Proceedings da Conferência Inovação ao Serviço da Saúde e do Doente; Março 2010; Lisboa; Apifarma; 2010.

14. Delgado AB, Lima ML. Contributo para a validação concorrente de uma medida de adesão aos tratamentos. Psicologia, Saúde e Doenças 2001; 2 (2): 81-100.

15. Departamento de Consolidação e Controle de Gestão do SNS. Estatística do movimento assistencial dos Centros de Saúde para o ano 2003. Lisboa: ACSS; 2003.

16. Briesacher BA, Gurwitz JH, Soumerai SB. Patients at-risk for cost-related medication nonadherence: a review of the literature. J Gen Intern Med 2007 Jun; 22 (6): 864-71. 
17. Westhoff CL, Torgal AT, Mayeda ER, Shimoni N, Stanczyk FZ, Pike MC. Predictors of noncompliance in an oral contraceptive clinical trial. Contraception 2012 May; 85 (5): 465-9.

18. Jirón M, Escobar L, Arriagada L, Orellana S, Castro A. Factores asociados al incumplimiento de los tratamientos con antidepresivos en Santiago, Chile. Value Health 2011 Jul-Ago; 14 (5 Suppl 1): S115-8.

19. Bugalho A, Carneiro AV. Intervenções para aumentar a adesão terapêutica em patologias crónicas. Norma de Orientação Clínica. Lisboa: Centro de Estudos de Medicina Baseada na Evidência da Faculdade de Medicina de Lisboa; 2004.

\section{CONFLITOS DE INTERESSE}

Os autores declaram não possuir qualquer tipo de conflitos de interesse.

\section{ENDEREÇO PARA CORRESPONDÊNCIA}

Ângela Maria Lee Chin

Rua dos Lusíadas N8 R/C Esquerdo

2625-435 Forte da Casa

E-mail: angelalee.chin@gmail.com

Recebido em 23/05/2012

Aceite para publicação em 15/10/2012

\section{ABSTRACT}

\section{INFLUENCE OF FINANCIAL FACTORS ON ADHERENCE TO MEDICATION}

Background: Financial factors are known to influence adherence to medication.

Objectives: To study adherence to medication, the different forms of cost-related non-adherence and the degree of reporting that information to the doctor, as well as to study measures to facilitate adherence.

Type of study: Cross-sectional study

Setting: Loures Family Health Unit

Population: Patients over 17 years of age in April 2011, registered with a family physician in the Loures Family Health Unit.

Methods: A convenience sample of 227 patients received the study questionnaire. It evaluated forms of non-adherence due to financial reasons in the past four months including increasing the time between doses, taking smaller doses, postponing the purchase of medication, and not purchasing medication. It also assessed if the patient had reported cost-related non-adherence to the doctor, non-adherence for other reasons, requests for less expensive drugs, requests for financial support for medication and delaying other purchases to buy medication.

Results: Of the 227 people studied, $79 \%$ reported medication non-adherence. Of these, 51\% were for financial reasons ( $31 \%$ exclusively). Cost-related non-adherence was expressed by $84 \%$ of patients by postponing the purchase of medication, in $46 \%$ by not purchasing medication, in $44 \%$ by increasing the time between doses and in $38 \%$ by taking smaller doses. Among those patients with cost-related non-adherence, only $30 \%$ reported it to their doctors. Regarding measures to facilitate medication adherence, $52 \%$ of patients said they have asked for less expensive drugs, $41 \%$ did not buy other things to purchase medication, and $12 \%$ asked for economic support to purchase medication.

Conclusions: Cost-related non-adherence was reported by a high percentage of individuals in this sample. Most patients did not report this to their doctors.

Keywords: Medication; Adherence; Financial Factors. 